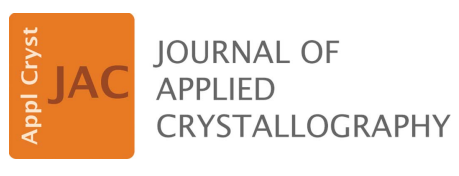

ISSN 1600-5767

Keywords: intragranular strain; $\mathrm{X}$-ray diffraction; 3DXRD; tomography.

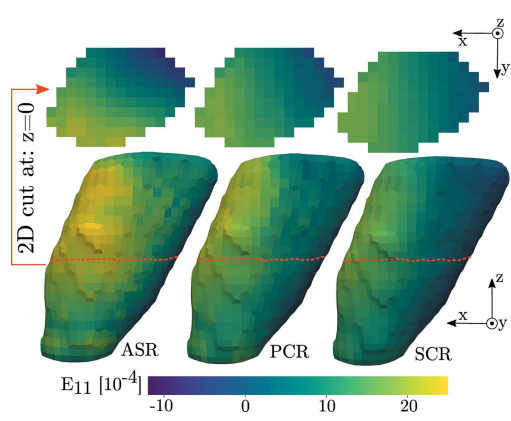

(C) 2020 International Union of Crystallography

\section{Strain tensor evaluation in polycrystalline materials by scanning high-energy $\mathrm{X}$-ray diffraction}

\author{
András Borbély* \\ Mines Saint-Etienne, Univ. Lyon, CNRS, UMR 5307 LGF, Centre SMS, F-42023 Saint-Etienne, France. *Correspondence \\ e-mail: andras.borbely@mines-stetienne.fr
}

During the past 20 years, third-generation high-energy synchrotron sources have made possible the development of several diffraction-imaging methods, which have led to meaningful physical insights into the real structure and dynamics of bulk polycrystalline materials. A not exhaustive list could include work on stress corrosion cracking (King et al., 2008), predictions of crystal plasticity (Pokharel et al., 2014), stress variation in copper through-Si vias (Levine et al., 2015), martensitic transformation (Sedmák et al., 2016) and long-range symmetry breaking in embedded ferroelectrics (Simons et al., 2018). The pioneering work of Poulsen (2004) paved the way for the majority of the techniques using monochromatic radiation, commonly called three-dimensional X-ray diffraction (3DXRD) or high-energy diffraction microscopy (HEDM) (Suter et al., 2006). Since then, a wide variety of techniques have been developed, which are mostly classified on the basis of the distance between the detector and the specimen as $(a)$ far-field or $(b)$ near-field techniques. However, from the point of view of the final reconstruction describing the spatial distribution of the crystallographic orientation (implicitly the grain shape) and strain/stress, a classification based on their spatial and angular resolutions seems to be more appropriate. According to this 'two-resolution criterion', all existing techniques can be included in one of the following three categories:

(a) High-spatial-and low-angular-resolution methods use a near-field setup with a highresolution detector (effective pixel size of about $1 \mu \mathrm{m}$ ), closely placed downstream of the specimen at a distance of a few millimetres. Their angular resolution is relatively low $\left(\sim 0.1^{\circ}\right)$, but they can resolve the structure at micrometre/sub-micrometre length scales over millimetre sample sizes. Grain and intragrain orientations are obtained via scans done with either a broad (Ludwig et al., 2008) or a planar beam (Li \& Suter, 2013), the beam size being usually adapted to the grain size to avoid peak overlap.

(b) Low-spatial- and high-angular-resolution methods involve a low-resolution detector with a pixel size of about $50-200 \mu \mathrm{m}$ placed in the far field $(\sim 0.2-1 \mathrm{~m}$ as a function of the pixel size and beam energy). Since diffraction peak positions can be determined with sub-pixel accuracy (Borbely et al., 2014), this setup has high angular resolution $\left(<0.01^{\circ}\right)$ that allows the elastic strain to be determined. However, the grain shape remains unknown. Measurements are usually performed with a broad beam.

(c) Finally, high-spatial-and high-angular-resolution methods enable characterization of both the crystallographic orientation and the strain inside single grains at submicrometre length scales, even for deformed materials. It is expected that these 'holy grail' methods will provide the missing local experimental evidence for understanding unsolved problems in materials science such as polycrystal plasticity, recrystallization and damage initiation. There are already two methods achieving high spatial and high angular resolution: differential-aperture X-ray microscopy (DAXM; Larson et al., 2002) and dark-field X-ray microscopy (DFXM; Simons et al., 2015). The first uses a polychromatic pencil beam with sub-micrometre cross section for identifying the local orientation from the resulting Laue pattern and an additional energy scan (Chung \& Ice, 1999) for strain determination. DFXM uses monochromatic radiation and compound refractive lenses in the diffracted beam, which magnify a small diffractive volume of the analyzed grain/ subgrain.

To improve the spatial resolution of the low-spatial- and high-angular-resolution methods, Hayashi et al. (2015) proposed a 'scanning 3DXRD' approach, using a monochromatic pencil beam, where the spatial resolution could be controlled by the beam size. Combining a lateral scanning of the sample and a tomographic approach (rotation 
around an axis perpendicular to the beam), the authors were able to reconstruct the variation of the crystallographic orientation inside single grains. Very recently, the method was also applied for determining local strain and stress tensors (Hektor et al., 2019; Hayshi et al., 2019). These developments, however, considered a simplified reconstruction procedure based on the assumption that the crystallographic structure in a given voxel can be refined from the subset of diffraction peaks generated by the beams that intersect its volume. Evidently, this assumption, which could be referred to as 'single-crystal reconstruction' (SCR), does not obey the tomographic principle, where the recorded signal is an average response over the X-ray beam path.

The paper of Henningsson et al. (2020) in this journal issue suggests two solutions to the tomographic problem of lattice strain, both considering all voxels in the beam path. The first, called 'polycrystal reconstruction' (PCR), is based on the minimization of the Euclidean distance between the experimental and simulated peak centers measured on the detector. The simulation approximates the scattered intensity by assuming that the contribution of a given voxel to the measured peak is proportional to the fraction of the voxel illuminated by the beam. The second solution is inspired by the common algebraic reconstruction (ART) method, as used, for example, by Alpers et al. (2006) to reconstruct grain shapes from 3DXRD data, extended to reconstruct tensor fields of smoothly varying intragranular strain. Both the PCR and ART methods were checked against simulated data and performed better than the simple SCR method. Finally, the strain state in a columnar tin grain was determined from experimental data measured at the ID11 beamline of the ESRF using a pencil beam with a cross section of $0.25 \times 0.25 \mu \mathrm{m}$. The reconstructions obtained with all three methods (SCR, PCR and ART) evidence the existence of a strain gradient in the grain.

By establishing the correct tomographic approach, the paper of Henningsson et al. (2020) upgrades 'scanning 3DXRD' to a viable alternative to DAXM and DFXM. Since it only involves lateral sample scanning and rotation around a single axis, the technique can be easily implemented in practice, which helps its wide use. However, to gain more confidence in the results, checking of real reconstructions against other techniques [such as near-field HEDM, similarly to Renversade et al. (2016)] would be valuable. Today, the scan duration for representative volumes is somewhat long $(12 \mathrm{~h}$ for scanning a volume of $37^{3} \mu \mathrm{m}^{3}$ with a beam size of $1 \times 1 \mu \mathrm{m}$ in steps of $1 \mu \mathrm{m}$; Hayasi et al., 2019). However, with the advent of fourth-generation synchrotron sources and fast detectors, the scanning time can be reduced by one order of magnitude, which already allows in situ studies to be performed during common beam time duration.

\section{References}

Alpers, A., Poulsen, H. F., Knudsen, E. \& Herman, G. T. (2006). J. Appl. Cryst. 39, 582-588.

Borbely, A., Renversade, L., Kenesei, P. \& Wright, J. (2014). J. Appl. Cryst. 47, 1042-1053.

Chung, J.-S. \& Ice, G. E. (1999). J. Appl. Phys. 86, 5249-5255.

Hayashi, Y., Hirose, Y. \& Seno, Y. (2015). J. Appl. Cryst. 48, 10941101.

Hayashi, Y., Setoyama, D., Hirose, Y., Yoshida, T. \& Kimura, H. (2019). Science, 366, 1492-1496.

Hektor, J., Hall, S. A., Henningsson, N. A., Engqvist, J., Ristinmaa, M., Lenrick, F. \& Wright, J. P. (2019). Materials, 12, 446.

Henningsson, N. A., Hall, S. A., Wright, J. P. \& Hektor, J. (2020). J. Appl. Cryst. 53, https://doi.org/10.1107/S1600576720001016.

King, A., Johnson, G., Engelberg, D., Ludwig, W. \& Marrow, J. (2008). Science, 321, 382-385.

Larson, B. C., Yang, W., Ice, G. E., Budai, J. D. \& Tischler, J. Z. (2002). Nature, 415, 887-890.

Levine, L. E., Okoro, C. \& Xu, R. (2015). IUCrJ, 2, 635-642.

Li, S. F. \& Suter, R. M. (2013). J. Appl. Cryst. 46, 512-524.

Ludwig, W., Schmidt, S., Lauridsen, E. M. \& Poulsen, H. F. (2008). J. Appl. Cryst. 41, 302-309.

Pokharel, R., Lind, J., Kanjarla, A. K., Lebensohn, R. A., Li, S. F., Kenesei, P., Suter, R. M. \& Rollett, A. D. (2014). Annu. Rev. Condens. Matter Phys. 5, 317-346.

Poulsen, H. F. (2004). Three-Dimensional X-ray Diffraction Microscopy: Mapping Polycrystals and Their Dynamics. Berlin, Heidelberg: Springer.

Renversade, L., Quey, R., Ludwig, W., Menasche, D., Maddali, S., Suter, R. M. \& Borbély, A. (2016). IUCrJ, 3, 32-42.

Sedmák, P., Pilch, J., Heller, L., Kopeček, J., Wright, J., Sedlák, P., Frost, M. \& Šittner, P. (2016). Science, 353, 559-562.

Simons, H., Haugen, A. B., Jakobsen, A. C., Schmidt, S., Stöhr, F., Majkut, M., Detlefs, C., Daniels, J. E., Damjanovic, D. \& Poulsen, H. F. (2018). Nat. Mater. 17, 814-819.

Simons, H., King, A., Ludwig, W., Detlefs, C., Pantleon, W., Schmidt, S., Stöhr, F., Snigireva, I., Snigirev, A. \& Poulsen, H. F. (2015). Nat. Commun. 6, 6098.

Suter, R. M., Hennessy, D., Xiao, C. \& Lienert, U. (2006). Rev. Sci. Instrum. 77, 123905. 